Article

\title{
Designing and Managing a Smart Parking System Using Wireless Sensor Networks
}

\author{
Adil Hilmani * iD, Abderrahim Maizate and Larbi Hassouni \\ RITM-ESTC/CED-ENSEM, University Hassan II, Km7, El jadida Street, B.P. 8012, Oasis, \\ Casablanca 8118, Morocco; maizate@hotmail.com (A.M.); lhassouni@hotmail.com (L.H.) \\ * Correspondence: adil.hilmani@ofppt.ma; Tel.: +212-633-33-22-12
}

Received: 10 April 2018; Accepted: 31 May 2018; Published: 6 June 2018

\begin{abstract}
For several years, the population of cities has continued to multiply at a rapid pace. The main cause of this phenomenon in developing countries like Morocco is the rural exodus. In fact, rural youth are increasingly attracted by the modern way of life and the opportunities of employment offered by cities. This increase in population density has a large number of negative effects on the quality of life in the city. The most obvious is the intensity of the traffic, which has become an almost insurmountable problem and which causes a great deal of damage, such as the increase in the number of accidents that cause serious bodily harm to the road users, the pollution caused by the large amount of $\mathrm{CO}_{2}$ released by the vehicles, and the continuous stress of drivers who must drive in often narrow and very busy roads and who must look for a long time to find a space to park. Thus, to solve the parking problem, several modern technologies have been created to equip car parks with smart devices that help road users identify the nearest car park that has a free space. These technologies most often use wireless sensor networks and Internet of Things (IoT) technology. In this paper, we present the design and development of a smart parking system using the latest technologies based on wireless sensor networks (WSN). Our system uses an adaptable and hybrid self-organization algorithm for wireless sensor networks that adapts to all types of car parks existing in the city (linear and mass parking), and offers a better management of the energy consumption during the wireless communication to increase the lifetime of the sensor nodes and the longevity of the WSN. This system also offers innovative services which facilitate the task to the drivers when looking for an available parking space in the city near their destination, in a fast and efficient manner.
\end{abstract}

Keywords: parking system; smart parking; wireless sensor networks; RFID (radio-frequency identification)

\section{Introduction}

With the change of the global economy and modern life, the Information and Communication Technologies (ICT) sector has experienced a vital acceleration in its process, to adapt at such change. Today, people spend most of their time outside of their home environments, they travel daily to work, and they frequently go shopping centres and attractions, without forgetting the displacements to the centre of the city. This certainly caused an imbalance in the daily mobility that led to the development of parking services to avoid unnecessary driving around the city centre to simply search for a parking space. This, on the one hand, causes additional carbon dioxide emissions and damages the environment of the city's ecosystem. On the other hand, it increases drivers' frustration and traffic congestion in the city, which will certainly cause traffic accidents. All of this degrades the experience of the modern city's ecosystem and has become a major challenge in the development of future smart parking systems. 
Smart parking systems are systems that manage the difficulty of parking in the city in public or private areas, using several recent technologies, including WSNs (wireless sensor networks) and RFID (radio frequency identification) [1-4]. These systems obtain information on the available parking spaces in a parking area using real-time data collection by the sensor nodes scattered in the parking area, which allows users to use the additional services implemented by these systems, such as the automated payment service compatible with mobile phones, so that people can reserve their parking space in advance. Figure 1 illustrates the general architecture of a smart parking system.

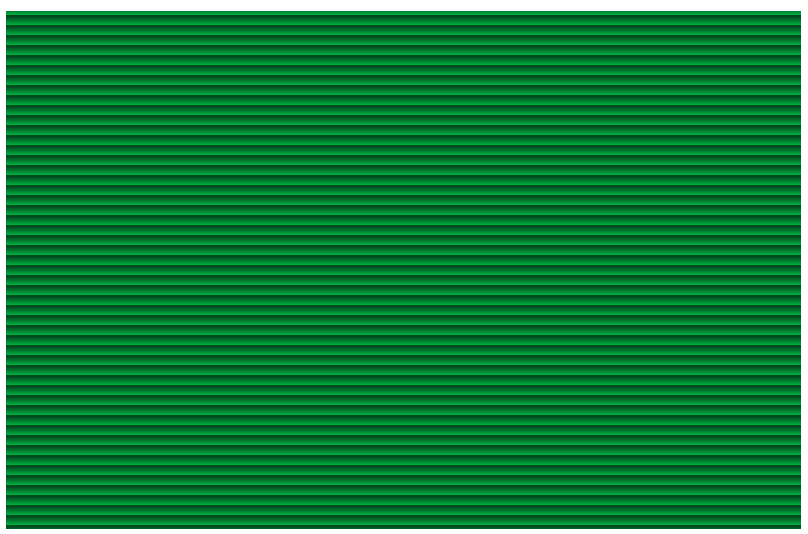

Figure 1. Smart parking system.

The system we propose in this article offers a solution based on an adaptable and hybrid self-organization algorithm for WSN networks, which allows to find a parking space in the two types of outdoor parking in a city. The first one is the "linear outdoor parking area" (Figure 2) which is a parking area located mainly on the main streets, alleys, and the centre of the city, where all parking spaces of the parking area form a single line. There are currently three types of linear outdoor parking areas (Figure 3). Slash-type parking is the easiest to park while the horizontal is the most difficult, followed by the vertical one. The second type is "Mass outdoor parking" which has a larger parking space than the linear car parks and are in the peripherals of the city and in larger areas like a technological park, shopping centre, etc. (Figure 4).

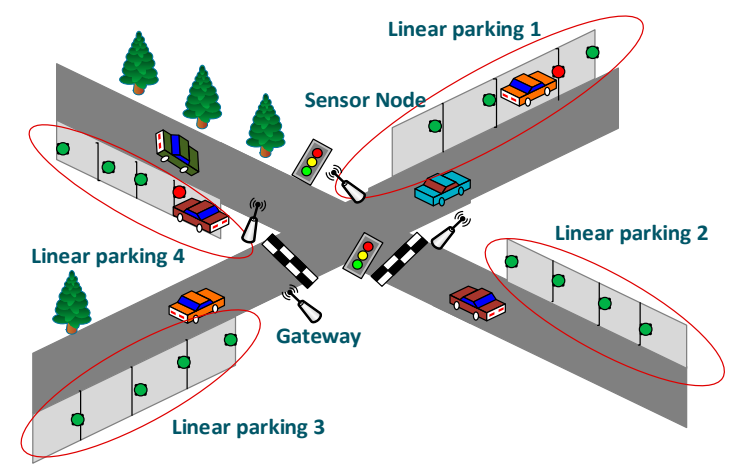

Figure 2. Linear outdoor parking.

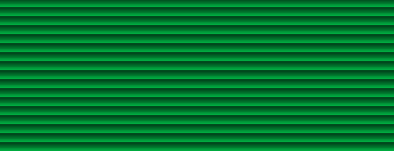

(a)

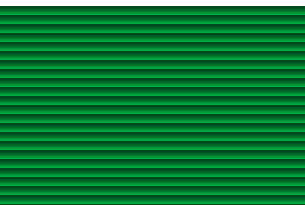

(b)

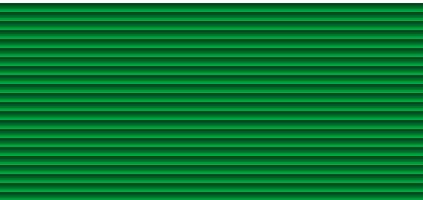

(c)

Figure 3. The types of linear outdoor parking (a) Horizontal type; (b) Vertical type; (c) Slash-type. 


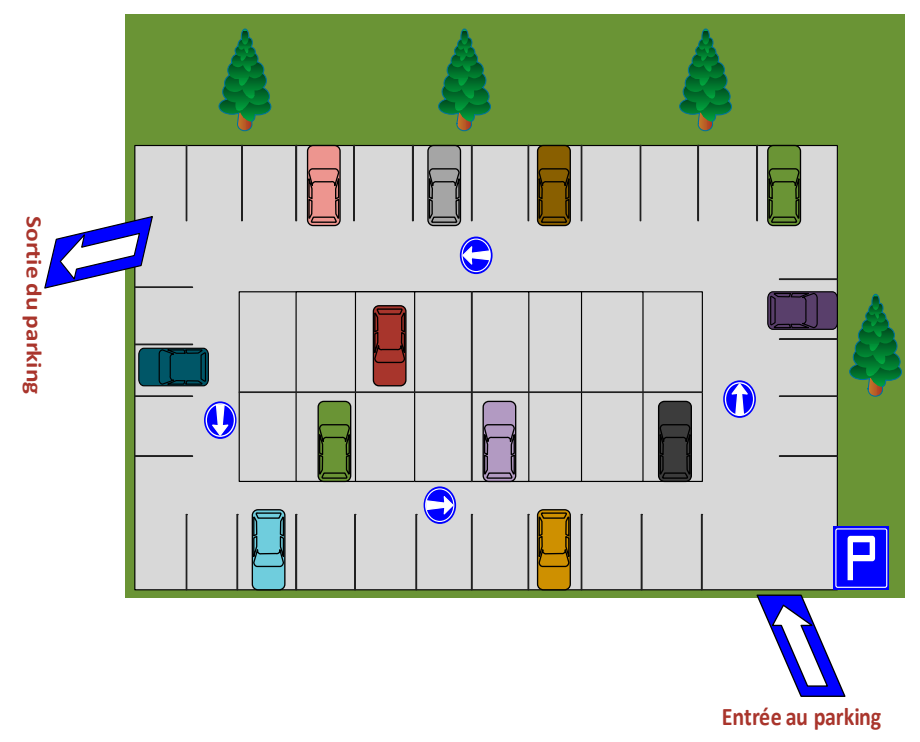

Figure 4. Mass outdoor parking.

Many smart parking management systems use self-organization algorithms for wireless sensor networks that are efficient and effective for a single type of parking (linear parking or mass parking), but they do not have the same performances for another type of parking. For example, for linear car parks, most of the self-organization algorithms used are based on chain formation, knowing that there are few sensors in the parking area, which allows minimizing the energy consumption between the different nodes and improving the energy efficiency in the network. However, this type of algorithm is not efficient and effective for mass parking knowing that there are many sensors in the car parking that introduce delays in the delivery of data to the gateway and that will create a load unbalance between the different nodes of the chain. For this reason, the self-organization algorithm for wireless sensor networks adopted by our system is adaptable for both types of parking (linear and mass) by forming chains or clusters according to the type, and which considers the limits of these networks in terms of energy consumption during the wireless communication process in order to increase the lifetime of the sensors and the longevity of the WSN network, by creating a powerful and sustainable system.

Our system also offers other very useful features, such as the management of parking spaces by identifying and checking vehicles parked in appropriate locations, the improvement of security against theft, the identification of available parking spaces near the destination of the drivers, and the control of the payment according to the duration of parking. The proposed system uses RFID technology (RFID readers and tags) to accomplish these tasks.

This system implements a web application and a mobile application to facilitate the task for drivers to quickly find a parking space at their destination on the one hand, and, on the other hand, for realize the payment of the duration of parking and effect online reservations in the case of private parking to make the system convenient for users.

The rest of the document is organized as follows: Section 2 provides an overview of the different types of WSN self-organization protocols used to manage the different types of car parks. In Section 3, we will propose the architecture of our car park management system based on WSN. Then, in Section 4, we will give a new contribution based on a proposed new smart parking management system using a hybrid self-organization protocol that can be adapted to any type of parking. After, in the Section 5, a comparison is presented to show advantages and disadvantages of each different smart parking systems and a comparison of technologies used in every system. Finally, we conclude with a perspective and a conclusion in Section 6. 


\section{Existing Smart Systems for Parking Management}

To manage and administer the various existing car parks in the city, there are two types of self-organization protocols for WSN: linear and mass.

\subsection{Linear Protocols}

Linear protocols are self-organization protocols used by wireless sensor nodes that are dispersed in an area of interest (in this case: parking areas) to form and construct a chain topology for collecting all the data detected and transfer them to the base station (Sink). Car park management systems that use these linear protocols include the following:

In [5], the proposed system is a system that uses two modules: a monitoring module and a reservation and security module. The surveillance module employs the network of wireless sensors to detect available spaces in a parking area, these sensor nodes are installed in each location forming a chain to collect information related to the states of the parking spaces. This information is sent to the car park management centre for efficient use towards the drivers (display of spaces, etc.). The reservation and security module uses the global system for mobile communication (GSM) system whose drivers must send an SMS to reserve their space in the parking. In return, the drivers receive a password with the number of the space in the parking area, so that they can enter and exit with all normality and with all security.

In [6], this article is based on the use of networks of wireless sensors whose nodes are infrared sensors that help to detect the presence of cars in parking spaces. The system consists of two modules: a monitoring module and a master module. The monitoring module consists of a ZigBee transmission and reception unit, a liquid-crystal display (LCD), and a peripheral interface controller (PIC) microcontroller which controls the data detected by the infrared sensor. Once the sensor detects the presence of a car, it informs the microcontroller, to display the status of this parking space on the LCD and, afterwards, it sends this data through the ZigBee transmission interface to the master module using a chain topology. The ZigBee technology used in data transmission shows a high performance in terms of energy consumption during wireless communication and is inexpensive to implement.

In [7], the system is based on the use of infrared sensors to detect the existence of cars in parking spaces. This system consists of three modules: the first module is the monitoring module which is responsible for the detection of parking spaces by the infrared sensors, and these sensors contain a PIC controller for data processing and a ZigBee system for data transmission using chain topology. The second module is the reservation module composed by a GSM module for the transmission and reception of data via SMS, allowing the reservation of parking spaces. The third module is the security module which uses the password already provided by the system allowing entry and exit only to authorized persons and who have an a priori reservation. The system of reservation based on GSM technology can be saturated with a high solicitation of the spaces of parking by the users, which can negatively affect the proper functioning of the parking system.

The proposed system CPF (Car Parking Framework) [8] manages a smart parking system that combines sensors (detection of parking spaces), RFID tags, and readers (parking access authorization, car's location, and prevention against thefts). This system uses a communication model based on the master/slave model between the sensor nodes in the bus mode with a serial cable communication. All nodes send their data to the master via cable, and afterwards it sends the detected data to the sink via a wireless communication. The installation of the serial cable for the communication between the nodes is expensive to implement and it limits the system for a future extension of the parking, which will cause complications in the development of the system.

In [9], the proposed system introduces a new smart parking system (SPS) based on the use of the latest technologies, such as WSNs, ZigBee, RFID, and NFC. The WSN constructs a topology in the form of a chain to collect information about available spaces in the parking area. This array of sensors consists of three types of sensors that communicate with the ZigBee technology. Sensor R detects the presence of a car in an available parking space, then it sends these data through various 
sensors $\mathrm{R}$ until it reaches sensor $\mathrm{C}$ (the smart gateway). Sensor $\mathrm{C}$ collects all the information and sends it to the central server. The RR sensors detect the RFID tags installed on cars authorized to park in special parking spaces. Once the label has been detected, this information is sent via multiple hops by nodes $\mathrm{R}$, until reaching node $\mathrm{C}$, which passes it to the central server. The SPS uses two applications to better manage the parking system, one for drivers, which helps them find parking spaces quickly and easily using the Google Maps navigation system with an online payment system (NFC). Another application, for authorities, allows them to receive alerts from the central server via GCM (Google Cloud Messaging) on unauthorized cars that have parked in the special spaces.

These linear protocols are not useful for the mass parking areas because these protocols will form longer chains which will create on the one hand delays in the delivery of data to the base station, and on the other hand, immense energy consumption during the transmission of the data. For this reason, mass protocols have been developed to deal with these problems in the efficient management of parking spaces in this type of parking area.

\subsection{Mass Protocols}

Mass protocols are self-organization protocols based on the deployment of WSNs in car parks allowing the creation of clusters or tree structures. The following systems use these protocols in car park management:

In [10], a new smart parking system (PGIS) is proposed, based on the use of networks of wireless sensors to control and manage parking by implementing a guidance system using LED screens. This system is based on a WSN which consists of three types of sensor nodes: a monitoring node (for detecting the presence of vehicles in parking spaces), a routing node (for routing and to route the detected information to the sink node), and the sink node (which gathers all information from the network to transfer it to the information and management centre). These nodes are installed in the car park and they form a tree topology. The PGIS uses a guidance system using LED screens that are installed at the entrance and in the corners of the car park so that drivers can orient themselves and find the assigned parking space efficiently and effectively. The PGIS information and management centre supports the management and maintenance of the entire system, processes the data collected by the monitoring nodes, calculates the optimal parking space for the new car, manages the parking costs of each parked car and controls all parking screens.

In [11], the author proposes a smart parking system that is based on the implementation of networks of wireless sensors for the management of vacant parking spaces for drivers who are looking for empty spaces. This system uses less expensive sensors (light sensors) to collect information on the status of parking spaces in order to send them to a central server through an aggregated server. The central server is installed and connected via the Wi-Fi network, and it receives all the information of all integrated web servers of each parking available in the city. This system uses a mobile application so that drivers can get information on vacant parking spaces. The light sensors used are influenced in most cases by ambient light and are sensitive to pollution, which can affect the quality and on the reliability of vehicle detection in parking spaces.

In [12], the author proposes a new smart parking system based on the implementation of various technologies, such as WSNs, RFID, and ZigBee. The network of wireless sensors is used to detect the presence of vehicles in the parking spaces with ultrasonic sensors installed in these spaces, and which inform the base station on the states of these locations (empty or full). These sensors are composed of two types of nodes: monitoring nodes that are installed in the parking locations, and the routing nodes that are responsible for transferring the information collected by the monitoring nodes to the base station by creating a tree topology. The communication between the various sensors and the base station is based on ZigBee technology which allows short distance communication and with reduced energy consumption. This parking system offers a new RFID technology that has a very important role in identifying vehicles which have just parked in an empty space and has a crucial role in pricing by controlling the time between check-in and check-out. 
In [13], the author has developed s smart parking system based on the use of hybrid wireless sensors (infrared sensors + RFID), which form a cluster-tree structure. Each of them is equipped with three LEDs (red, green, blue) to control and manage the availability of parking spaces. This proposed system consists of four essential modules that are the online booking module, the input module, the output module, and the parking management module.

In [14], the author created a new smart parking system (SIMERT) based on the installation of two wireless sensors in each parking space to detect the presence of vehicles and to control the good parking of vehicles in the parking spaces. This system uses WSN technology composed of three types of sensors (surveillance sensor nodes, routing nodes, and a sink node) that form a cluster topology. The parking space is divided into areas (clusters) where each agent is responsible for each area. This system implements two Android applications, one for drivers to see the available parking spaces, and the other for agents, whose system sends alerts on any event that occurs in the parking area of each agent.

In [15], the author implemented wireless network sensors to monitor and manage parking spaces in a parking lot. To detect the presence of vehicles, the author used proximity sensors consisting of an Arduino Uno which sends the data to a Raspberry Pi which is configured as a client (Sink) and which, in turn, sends the data received to the back-end server via Wi-Fi by creating a tree topology. This system uses a mobile application based on the data stored in this server to inform and locate the available parking areas closest to the destination of the drivers. The author has improved the system by adding a payment system using RFID tags that will be checked at the entrance of the car parks to realize the correspondence between RFID ID and the reserved space, and in the output, to realize the payment efficiently and quickly on the one hand, and on the other hand, to control the flight of cars in this parking area.

The Smart Parking Management System proposed in [16] is based on the implementation of three types of technologies used in the IoT domain, radio frequency identification (RFID), automatic license plate recognition (ALPR), and a wireless sensor network (WSN). The wireless sensor network executes a mass algorithm that allows the creation of a cluster topology between the different nodes, these sensor nodes are divided into three types. The SN nodes are in each space in a parking area to detect the availability of this space. The GN nodes are strategically located in the parking area to collect occupancy states transmitted by a defined number of SN nodes for transfer them to the CN node. The $\mathrm{CN}$ node is responsible to manage all information of the entire parking lot area and communicate it to a database server. This system uses additional services such as a mobile application to receive real-time updates and NFC (near-field communication) for the reservation of parking space and the online payment.

In [17], the author has developed a smart parking system based on the use of the wireless sensor network and IoT to manage the parking problem in outdoor car parks in the city. These nodes are installed in each parking space, and they are composed of an ultrasound sensor to detect the presence of a vehicle in the parking space and a Wi-Fi communication module to send the state of occupation to a server Cloud that manages all the parking spaces in the city. This WSN network implemented in this system does not form any network topology between the different sensor nodes to transfer the detected data to the cloud server. The stored data in the cloud server is exploited using an Android application (ParkX) so that users can consult and navigate to available parking spaces near their destinations. Wi-Fi wireless communication consumes a great deal of energy during data transmission, which will negatively affect the battery lifetime of the nodes and on the longevity of the network. However, this technology shows excellent performance in terms of obstacle penetration compared to ZigBee.

The author of [18] proposes a smart parking management system to manage the parking problem in mass parking in areas like an airport or a campus using RFID. The RFID readers are installed in each parking space and at the entrance and exit to secure the parking area and manage the availability states of parking spaces. The RFID readers activate the passive RFID tag on the card of the driver entering or exiting or parking the car in the area and read the information of the tag, which contains a unique 
identification number, to transfer them to the database of the system via a multi-hop communication using Wi-Fi technology, to update the occupation states of the parking spaces in real-time.

These mass protocols have improved delays in the delivery of the data to the base station and they downplayed the enormous energy consumption when transmitting this data compared to the linear protocols. However, these protocols are only applicable for car parks with a group or mass structure, which makes it possible to create clusters that are not useful for linear car parks of which they will create, on the one hand, a load imbalance between the sensor nodes and, on the other hand, minimize the lifetime of the entire network.

\section{Architecture of the Smart Parking System}

The proposed system contains three essential parts: parking detection centre, parking monitoring centre, and global information management centre.

- $\quad$ The parking detection centre is composed mainly of hybrid sensor nodes (sensors + RFID readers) that are installed in each parking space in each area, these sensor nodes form a wireless sensor network (WSN) allowing to collect the states of all the parking spaces (available or occupied) to send them to the gateway (Sink) of this area, this information will be sent afterwards to the central server to store them in the global database.

- The parking monitoring centre is responsible for identifying and checking the cars that have just parked in a reserved or available space. This centre uses the RFID technology to control and monitor the one hand the parked cars, and the other hand, for identify and manage the payment of parking time.

- The global information management centre is a database where all information detected and collected from all car parks in the city is recorded and exploited in real-time by web or mobile applications. In this way, drivers will have all the information on the available spaces in all the car parks of the city, to consult these spaces according to their destination, and to pay the parking fees.

Figure 5 shows the architecture of the proposed smart parking system:

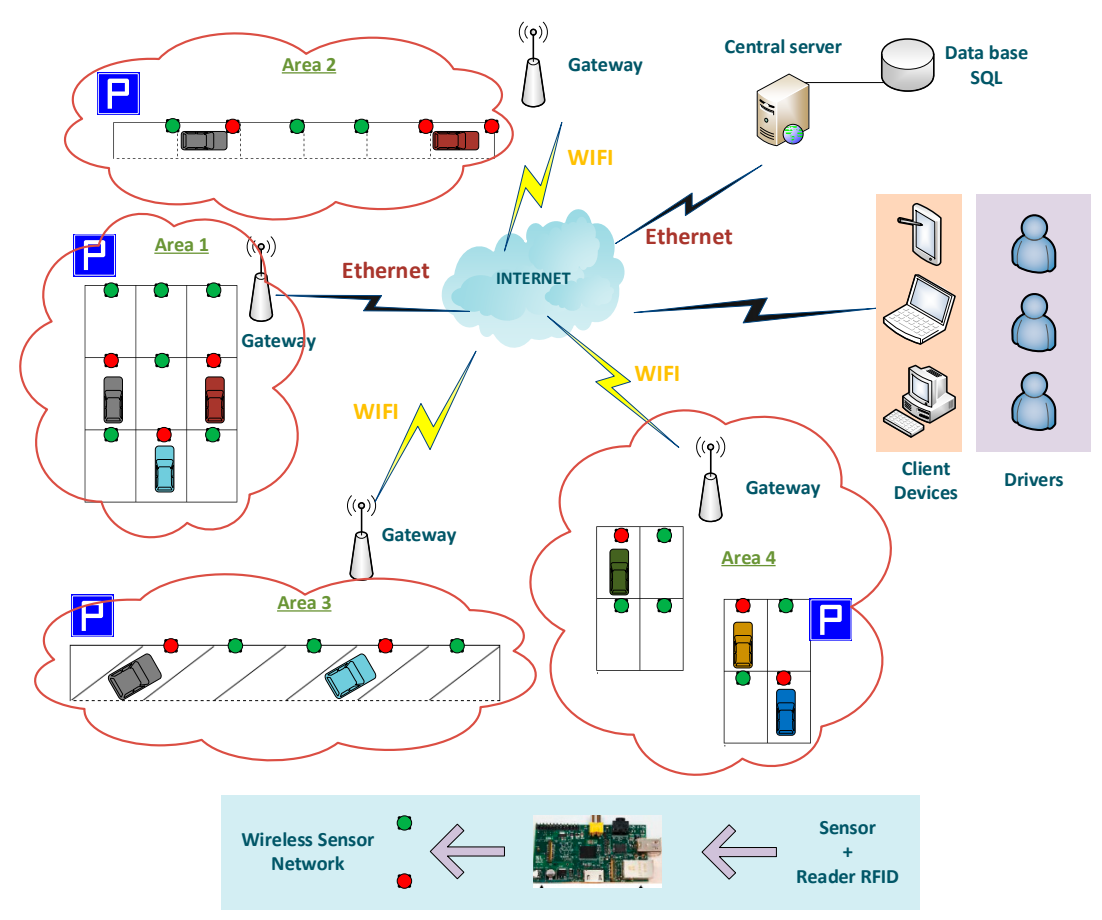

Figure 5. The proposed architecture of smart parking systems based on a WSN. 


\section{Proposed Smart System for Parking Management}

\subsection{Parking Detection Centre}

The parking detection centre uses two very recent technologies, wireless sensor networks (WSN) and RFID technology. The formation of the sensor network changes according to the type of parking in the area. For linear car parks, a chain topology will be formed in the network and, on the other side, a network topology in cluster form will be created in mass car parks. The formation of different topologies is based on the execution of a hybrid self-organization algorithm that is adaptable to the type and structure of parking that allows to form either a chain (Figure 6) or clusters (Figure 7), according to the distribution of the nodes and how they are scattered in the parking area.

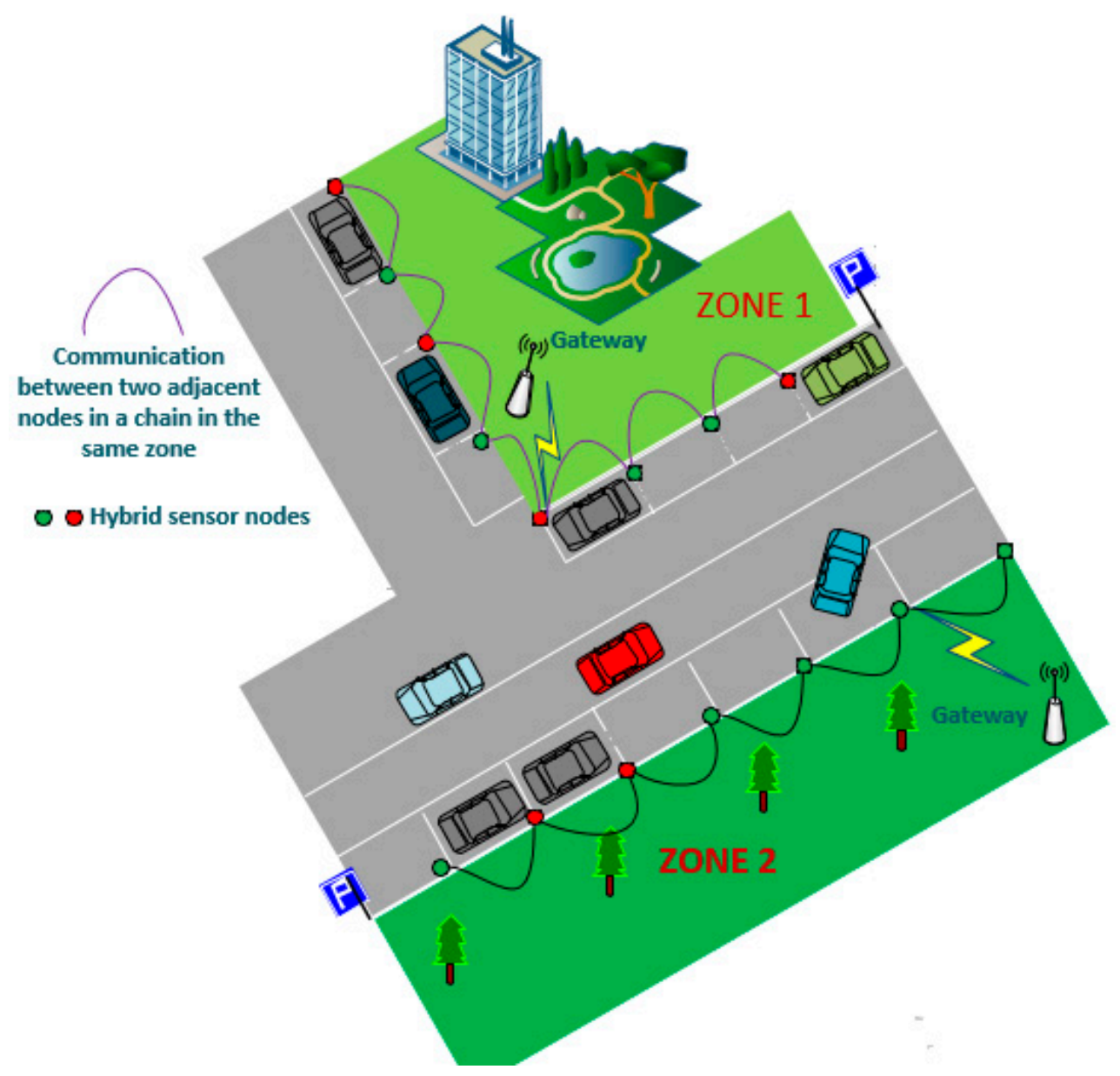

Figure 6. Formation of the chain topology in the linear parking. 


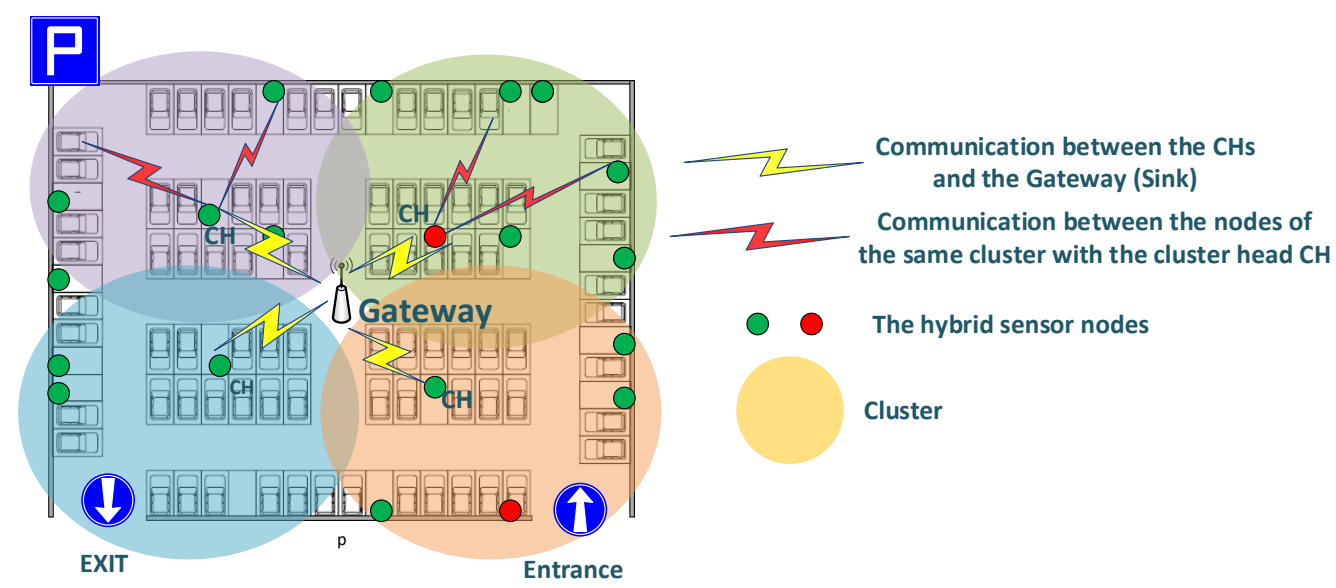

Figure 7. Formation of cluster topology in the mass parking area.

To form a network topology by the sensor nodes in an outdoor parking, all the nodes send their coordinates to the gateway which executes Algorithm 1 making it possible to calculate and detect the type of topology to be formed. In the case of linear parking, the gateway detects that all the nodes have the same $\mathrm{X}$ coordinate or the same $\mathrm{Y}$ coordinate as a function of the distribution of the nodes in the parking area. In this case, the gateway requests the nodes to create a chain topology in the network to minimize power consumption between the nodes using the multi-hop communication to the gateway (Figure 8).

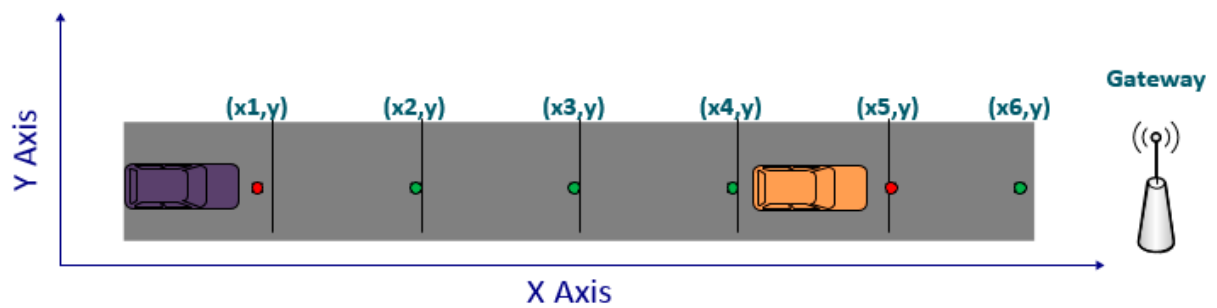

Figure 8. Example of a distribution of the sensor nodes in a linear parking area.

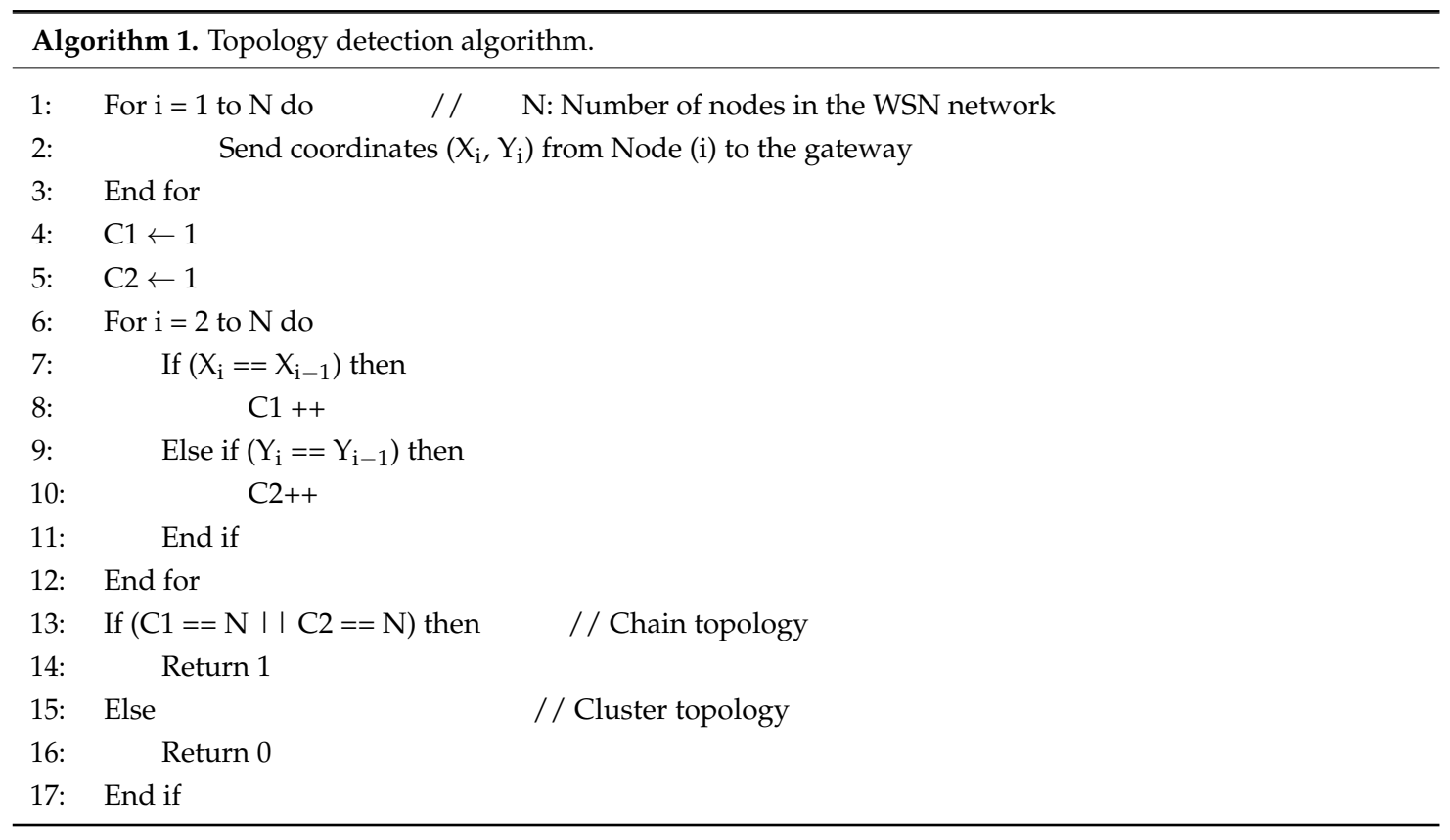


In the contrary case (mass parking), the gateway detects that all the nodes do not have similitude in one of the coordinate parameters (X or Y), so it will order the nodes to form a cluster topology to increase the longevity of the network knowing that, in this case, there are a large number of nodes scattered in the parking area (Figure 9).

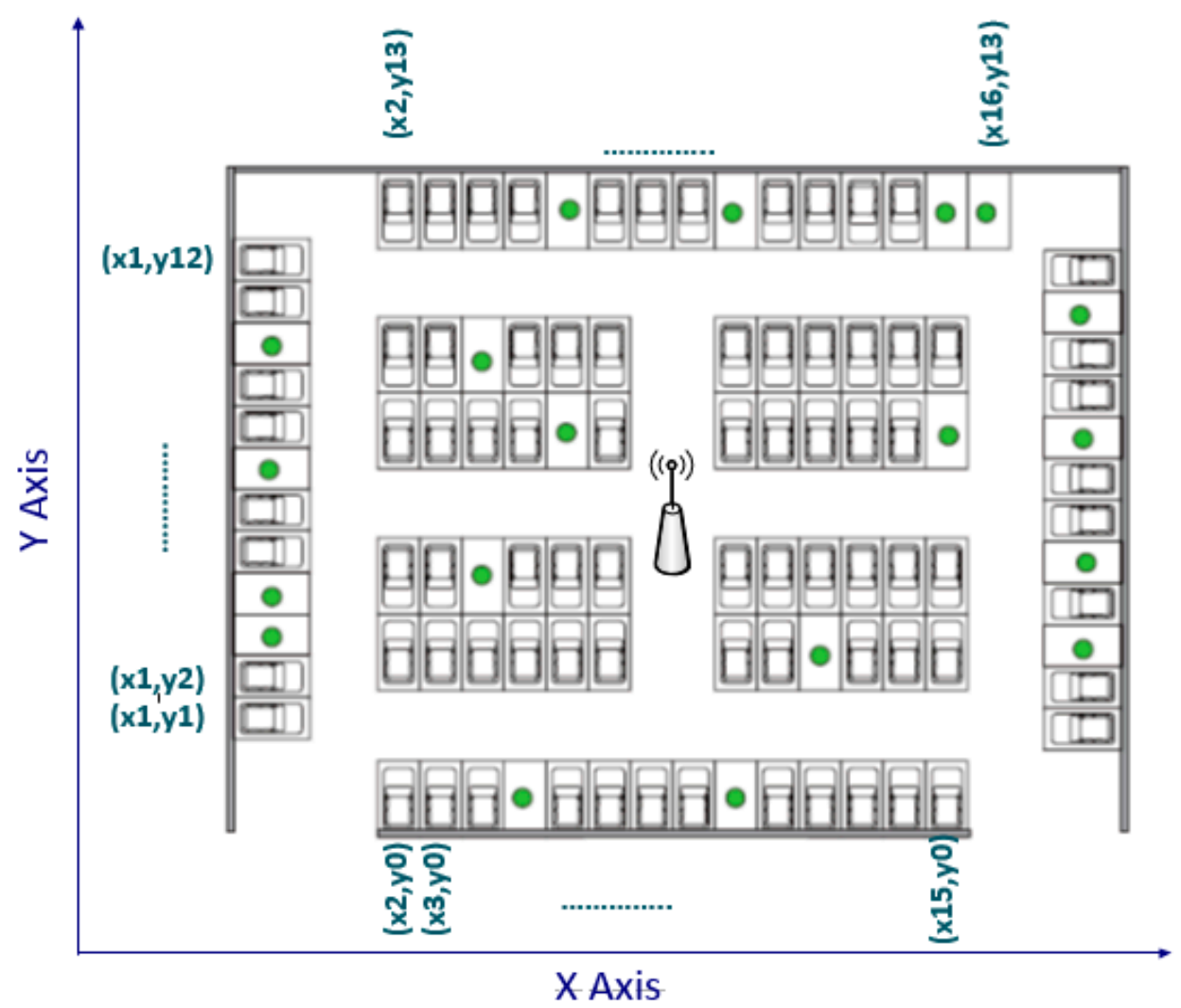

Figure 9. Example of a distribution of the sensor nodes in a mass parking area.

After the execution of the adaptable self-organization algorithm (Algorithm 2), a chain of the sensor nodes will be formed in the city's linear car parks, where all the nodes dispersed in each parking area will send their detected data via multiple hops to the node leader, who will gather and transfer all data from the parking to the gateway of each parking area. In the case of mass car parks, a cluster topology will be created in each parking area in the city where all sensor nodes will form clusters and in each cluster a $\mathrm{CH}$ will be elected to receive the detected data from all nodes in its cluster, and transfer them via a single hop to the Gateway (sink).

The formation of the chain or the formation of clusters will be based on the residual energy of the nodes and the overall energy of the network to maximize the lifetime of the nodes and increase the longevity of the network WSN. 


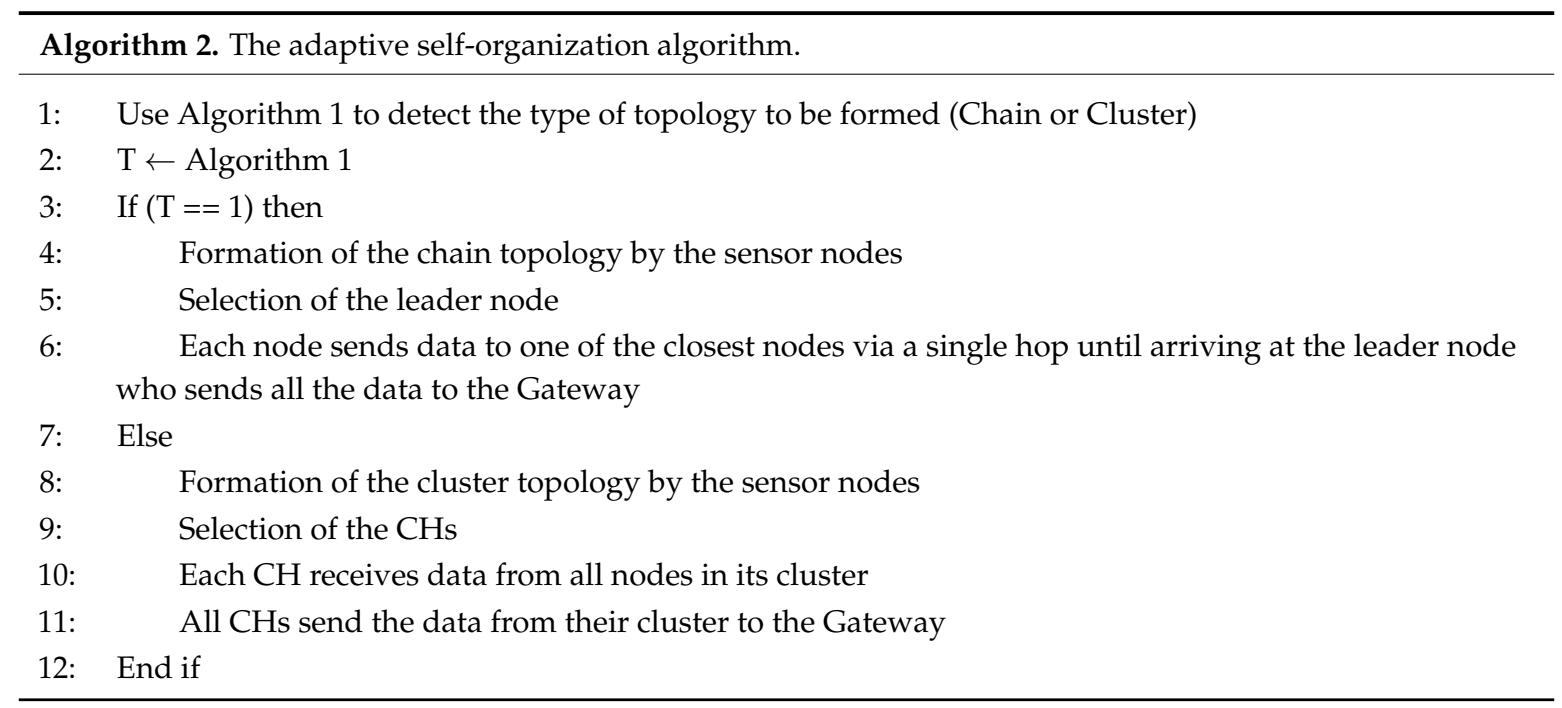

The sensor nodes are installed in each parking space and in each of the parking areas, which makes it possible to detect the presence or the absence of the vehicles in the latter. Each parking area (parking) is identified by an IDzi, and each parking space in a parking area is identified by IDpj, so each sensor node is identified by two pairs of the identifiers \{IDzi, IDpj\}. Once a car has just parked, the sensor node detects the presence of the latter and sends the occupied state of this space with both identifiers to the gateway. Afterwards, this information will be sent and saved in the central server.

To better illustrate the process of managing parking spaces in different parking areas, Algorithm 3 shows the operations performed by the sensor nodes in each parking area.

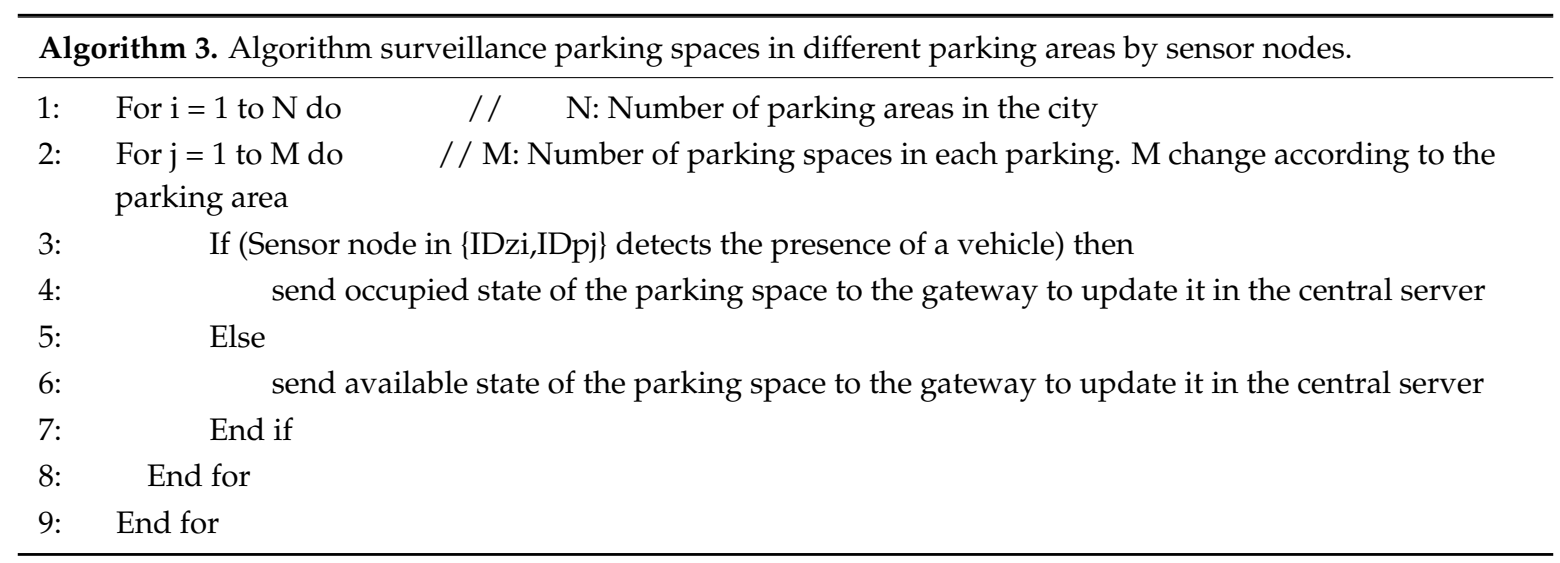

The wireless communication between the different sensor nodes and the gateway acts a crucial role in the deployment of the WSN network in the outdoor car parks, where much of the energy consumed by the nodes is highly dependent on the energy consumed during wireless communication:

$$
\begin{gathered}
E_{\text {Sensor }}=E_{\text {Sens }}+E_{\text {Proc }}+E_{\text {Comm }} \\
E_{\text {Sensor }} \approx E_{\text {Comm }}
\end{gathered}
$$

where $E_{\text {Sens }}$ is the energy consumed during the detection process, $E_{\text {Proc }}$ is the energy consumed during the treatment process, and $E_{\text {Comm }}$ is the energy consumed during the communication process.

To detect the availability of parking spaces in an outdoor parking lot in an efficient and reliable manner, the system must deploy one of the most used wireless communications technologies (Wi-Fi, Bluetooth, ZigBee, etc.), so to minimize the energy consumption of the nodes and to increase the longevity of the WSN network. The networks based on ZigBee technology generally consume less energy compared to Wi-Fi and Bluetooth networks. 
The following table (Table 1) shows the advantages of ZigBee over other wireless communications technologies.

Table 1. The advantages of ZigBee over other wireless communications technologies.

\begin{tabular}{ccccc}
\hline Wireless Parameter & Bluetooth & IEEE 802.11 b & IEEE 802.11 ah & ZigBee \\
\hline Frequency band & $2.4 \mathrm{GHz}$ & $2.4 \mathrm{GHz}$ & Sub-1 GHz & $2.4 \mathrm{GHz}$ \\
\hline Range & $9 \mathrm{~m}$ & 75 to $90 \mathrm{~m}$ & $\begin{array}{c}1000 \mathrm{~m} \text { (without } \\
\text { repeaters) }\end{array}$ & $\begin{array}{c}100 \mathrm{~m} \text { (without } \\
\text { repeaters) }\end{array}$ \\
\hline Current Consumption & $60 \mathrm{~mA}$ (Tx mode) & $\begin{array}{c}400 \mathrm{~mA} \text { (Tx mode) } \\
20 \mathrm{~mA} \text { (Standby mode) }\end{array}$ & $\begin{array}{c}90-140 \mathrm{~mA} \text { (Tx mode) } \\
5 \mu \mathrm{A} \text { (Standby mode) }\end{array}$ & $\begin{array}{c}25-35 \mathrm{~mA} \text { (Tx mode) } \\
3 \mu \mathrm{A} \text { (Standby mode) }\end{array}$ \\
\hline
\end{tabular}

On the other hand, the deployment of the WSN sensor network in outdoor car parks obliges sensor nodes to be installed in the ground, where the signal propagation is obstructed by cars and is affected by their noises. For this type of application, many previous works have shown that conventional wireless standards, such as Bluetooth or $\mathrm{Wi}-\mathrm{Fi}$, are not suitable for this type of network in terms of quality and performance compared to the ZigBee standard based on some parameters such BER (bit error rate) and SNR (signal-to-noise ratio) [19-21]. The following figure demonstrates the performance of ZigBee compared to other types of networks (Figure 10).

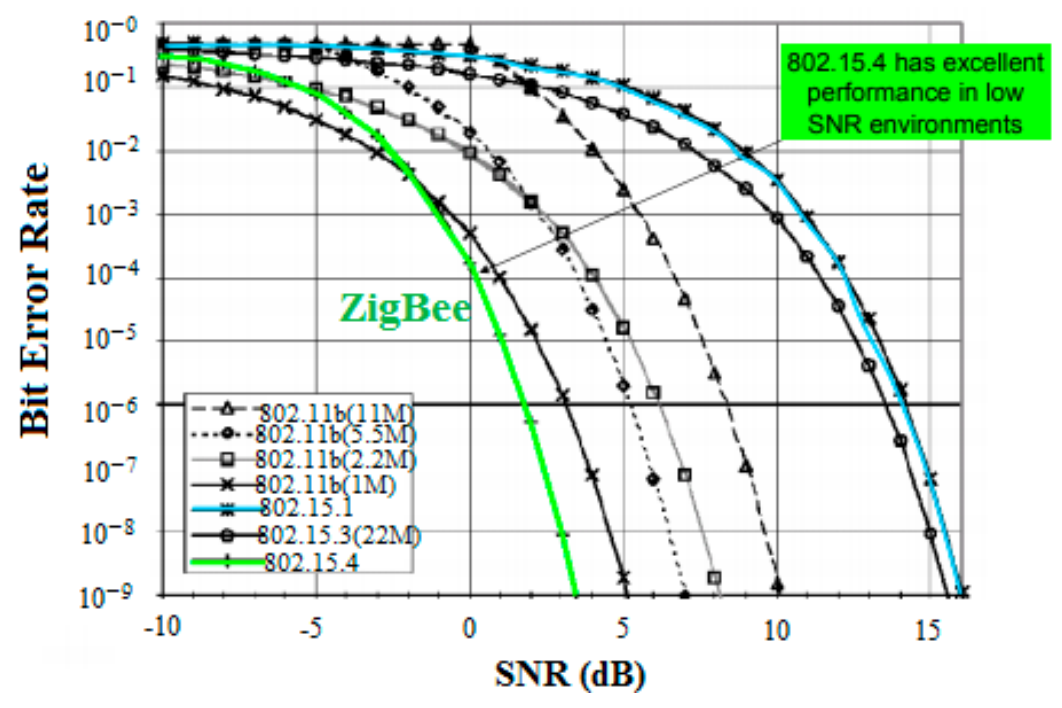

Figure 10. 802.15.4 ZigBee performance based on BER-SNR.

For this reason, we will use ZigBee wireless communication technology, adopted by the proposed self-organization protocol, to extend the battery lifetime of the sensor nodes and to increase the longevity, efficiency, and performances of the WSN network in the outdoor car parks.

\subsection{Parking Monotoring Center}

The parking monitoring centre uses wireless sensor networks (WSN) and integrated RFID technology in these sensor nodes installed in each parking space. RFID technology is a technology based on radio frequency identification that helps to check and identify objects by radio waves. Thus, vehicles will be identified and parking fees will be collected via this system, to manage and monitor the parking area in an efficient and convenient manner.

Once a car has just parked in a space $i$ in a parking area $j$, the appropriate sensor detects the presence of the vehicle and it sends the two identifiers \{IDzi, IDpj\} with the occupied state of that space to the gateway, to transfer them to the central server in order to store them in the database. The RFID reader, integrated in each sensor node, reads the driver's data using the RFID tag installed in the 
vehicle (name, first name, vehicle number, telephone number, etc.) and transfers them by merging them with the state of the space towards the central server. This server will be used to calculate the duration of parking and to control the detected incidents during the parking of a vehicle. In the case of cars without any RFID tag, a message is sent to the parking officers with the two parking identifiers $\{$ IDzi, IDpj\}, to register the vehicle registration number into the system to guarantee the payment of parking fees. Algorithm 4 illustrates the process of managing the parking space in each area by the central server, when a vehicle arrives at the parking area.

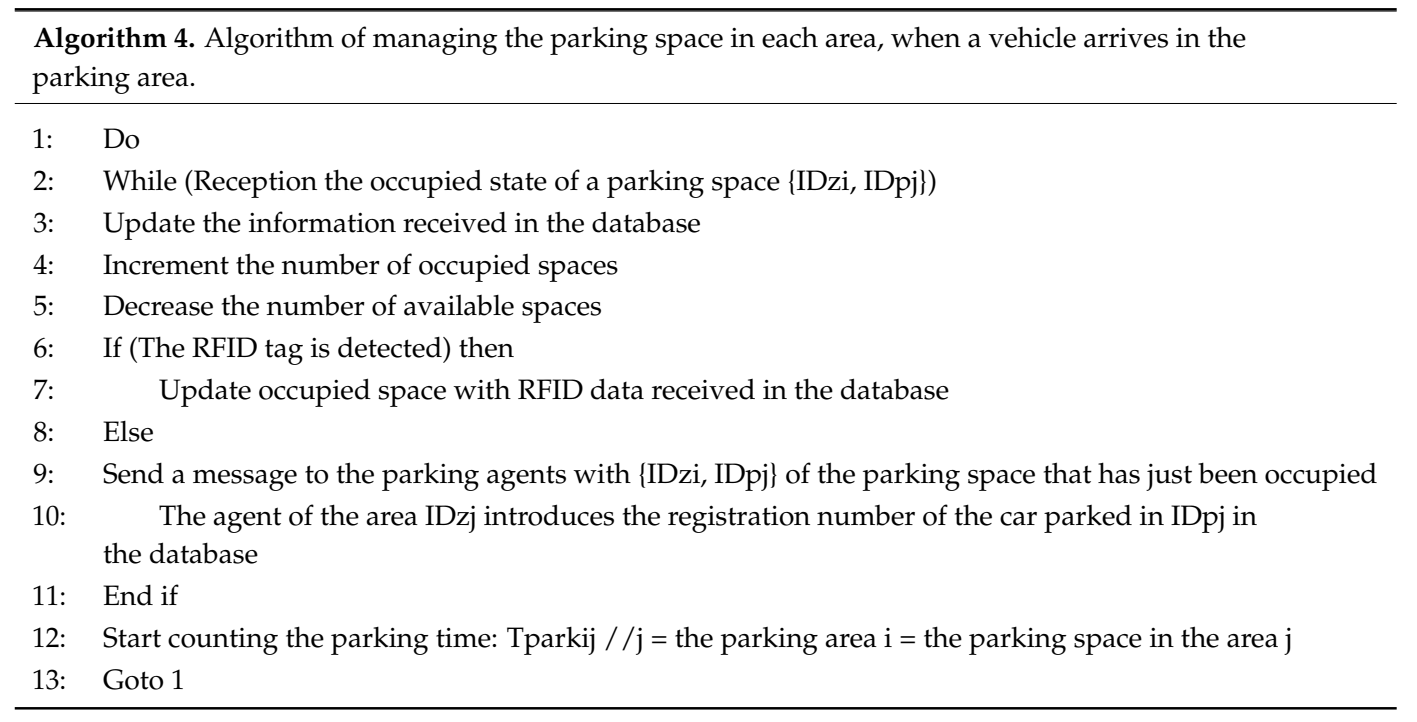

Once the car leaves the parking space, the sensor concerned detects the availability of the space by sending the two identifiers $\{\mathrm{IDzi}, \mathrm{IDpj}\}$ to the central server through the gateway so that it updates the state of the space in the database. After release of the space in the database the system checks whether the driver has made the payment for the duration of parking, otherwise a notice of infraction and a fine will be sent to the authorities after one hour so that the parking fees will be paid by the drivers.

The payment of the parking time is made online using the mobile application or manually in the automated teller machine in each area. In both cases, only the registration number of the parked vehicle must be entered so that the system can detect it in the database and make the payment. The algorithm below (Algorithm 5) shows the operations performed for the management of the parking spaces in each area when a car leaves the parking area.

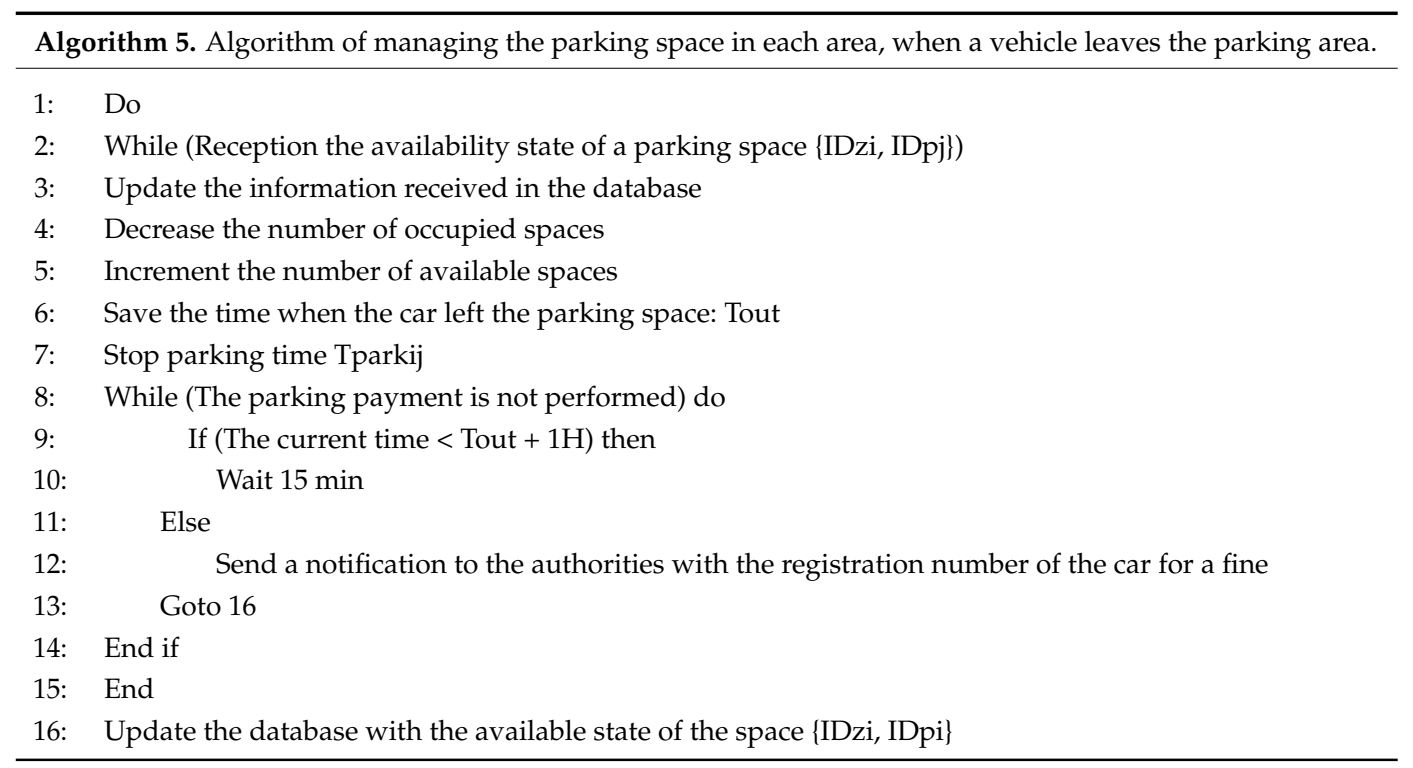




\subsection{Global Information Management Centre}

The global information management centre relies on the data stored in the database to develop extra services to the users and drivers by facilitating the task of searching for an available space in their destination, such as the consultation of the available parking spaces, the navigation to these spaces, and also the online payment of parking fees. A mobile application is developed that allows drivers to take advantage of these services in a practical and simple manner.

- $\quad$ Consultation of parking areas: Drivers use the mobile application which allows them to consult and find open parking spaces near their destination, before moving to avoid unnecessary travel and also not to create congestion of traffic. This application uses the information that is stored in the database to make available to drivers, the open parking areas closest their destination with the number of available and busy spaces in real-time, by indicating the parking price (Figure 11). Navigation to parking areas: This service is based on the use of Google MAP to orient and guide drivers towards the desired parking areas. The driver opens the mobile application to look for an open parking near his destination. Then, depending on the results displayed by the application, the driver selects a parking area and the app opens Google MAP to guide the driver to the selected area. Once arriving in the area, the application displays the available spaces and busy squares in this parking area. Figure 12 illustrates the operation of navigation to the parking areas. Payment of parking fees: Before leaving the parking space, the driver must pay the parking fees, this payment is made either manually by moving to the automated teller machine of the parking lot by entering the number of vehicle registration. Either online, the driver uses the same mobile application with the same manipulations of the automatic teller machine to realize the payment of the parking fees in this parking area.
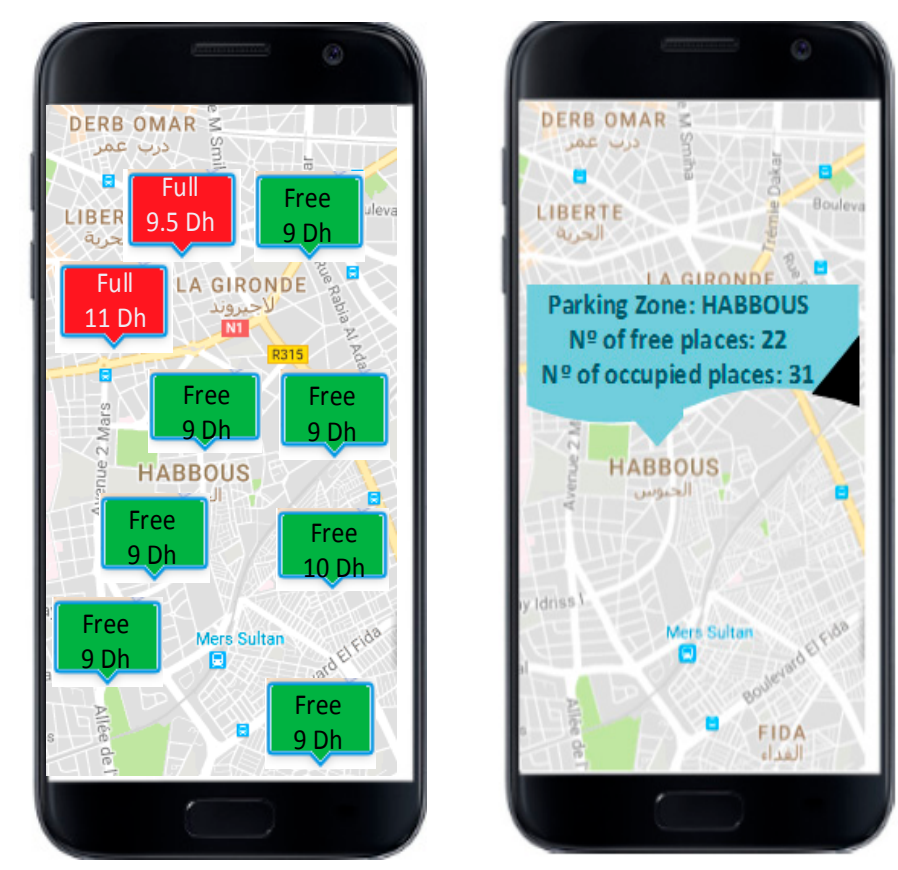

Figure 11. The consultation of the areas and the prices of parking. 


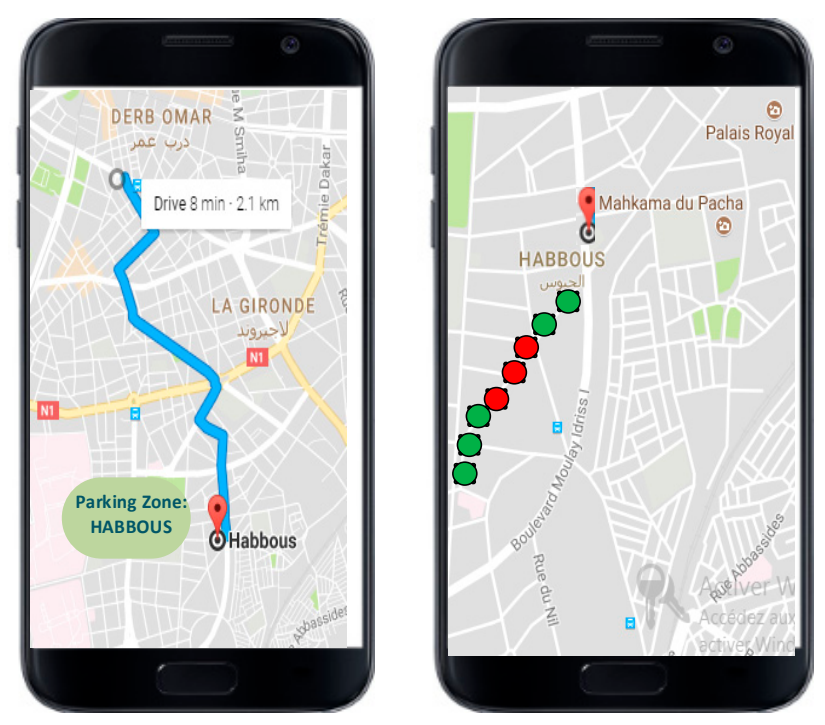

Figure 12. Navigation to a parking area.

Algorithms 6 and 7 shows the operation of the global information management centre of our smart parking system.
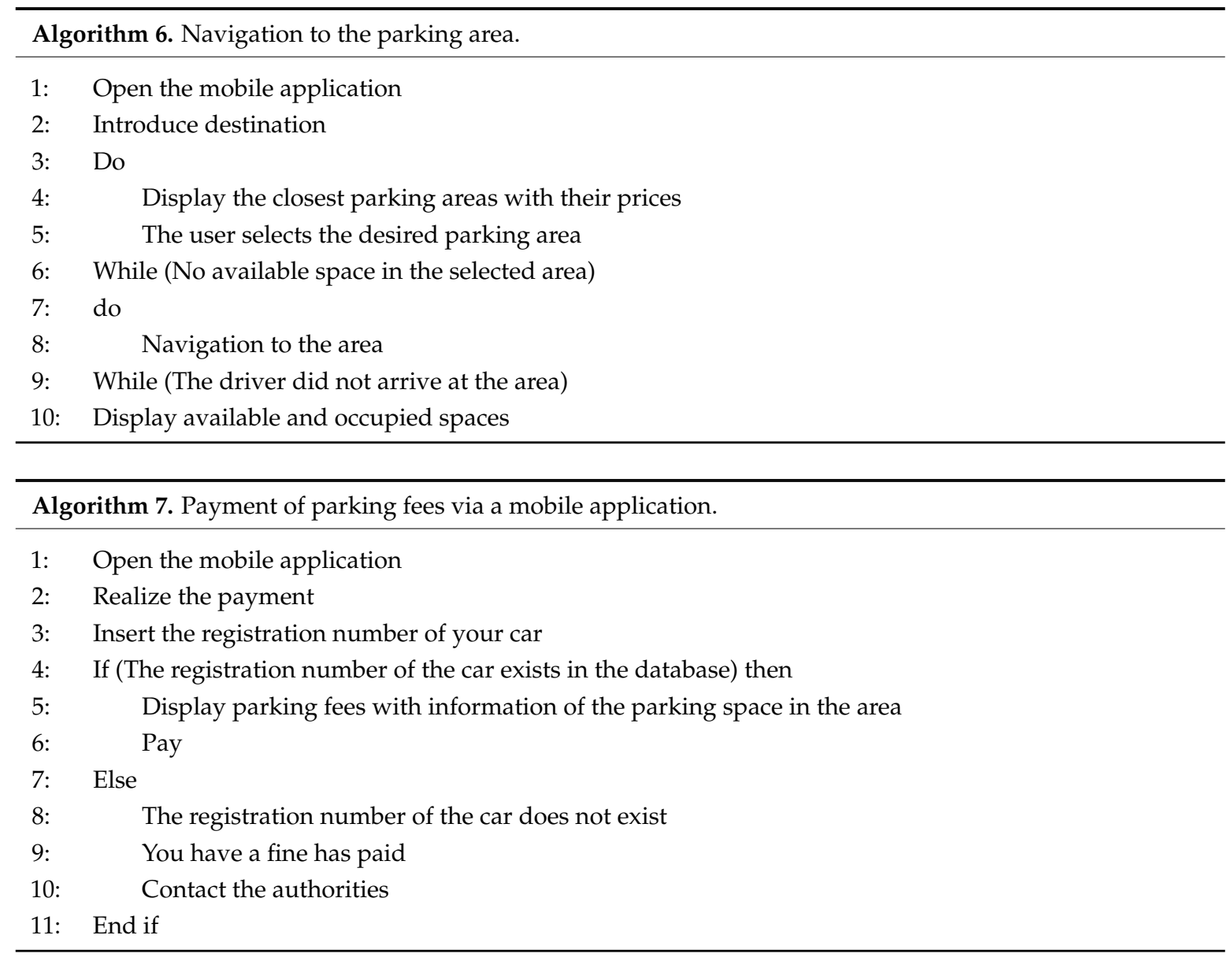

\section{Discussion}

According to the different architectures of smart parking system studied in this article, Tables 2 and 3 summarize the advantages, disadvantages, technologies, and services used by these 
architectures. There is no system that adapts to the different types of architectures and structures of parking (linear and mass). There are parking systems that only work for linear car parks and do not have the same impact at the deployment level of sensor networks in mass parking, and vice versa.

The complexity of the algorithms used in the management of parking spaces plays an important and crucial role in the development and design of a robust and efficient smart parking system. The majority of the algorithms proposed by our system are not complex, they are easy to be implemented, and they are executed at the server level, which minimizes the exhaustion of the sensors and does not overload them, so that they do not consume a large amount of energy in order to increase the duration of the lifetime of their batteries.

Energy efficiency is one of the important parameters for many self-organization algorithms that allows the creation of a solid network topology in order to optimize the energy consumption between the different sensor nodes and to increase the longevity of the WSN network. The self-organization algorithm adopted by our system is a flexible and unique algorithm, compared to the algorithms implemented by the smart parking systems studied in this article, which allows the creation of a chain topology for linear parking areas and a cluster topology for mass parking areas, in order to balance the load between the different sensors and minimize the energy consumption during the transmission of data to obtain a better energy efficiency management and increased the lifetime of the nodes.

For this reason, we will offer an adaptable smart parking system that allows for the creation of flexible WSN network topologies for any type of existing parking in the city, based on many services and technologies offering convenience to the driver. Systems technology with an adaptable wireless sensor network (WSN) architecture provides a flexibility and a suppleness in the deployment of smart parking systems that will be monotonous in the design and implementation, and will also be standardized in the development of applications and services for the different types and structures of existing car parks, bearing in mind that this solution creates a solid basis for the development and improvement of these systems in the future, as required.

The smart parking system proposed in this article is based on the implementation of two most recent technologies, such as WSN and RFID. The use of these two technologies only gives a plus compared to other parking management systems in terms of the implementation cost and also in terms of design quality. 
Table 2. Advantages and disadvantages of different smart parking system.

\begin{tabular}{|c|c|c|c|}
\hline $\begin{array}{l}\text { Smart Parking } \\
\text { Systems }\end{array}$ & $\begin{array}{c}\text { Type of Parking } \\
\text { System }\end{array}$ & Advantages & Disadvantages \\
\hline S1: [5] & $\begin{array}{l}\text { Interior parking, } \\
\text { linear, no adaptable, } \\
\text { single area }\end{array}$ & $\begin{array}{ll}\text { - } & \text { Flexible and easy system implemented. } \\
\text { - } & \text { System is private with the spaces reservation via GSM before moving. } \\
\text { - } & \text { Less expensive system. } \\
\text { - Security with a password received by the GSM. }\end{array}$ & $\begin{array}{ll}\text { - } & \text { No application is developed for remote monitoring. } \\
\text { - } & \text { Access only to the driver who made a reservation a priori. } \\
\text { The blocking of the GSM system during mass access to the system. } & \text { No guidance and navigation system is used. }\end{array}$ \\
\hline S2: [6] & $\begin{array}{l}\text { Interior parking, } \\
\text { linear, no adaptable, } \\
\text { single area }\end{array}$ & $\begin{array}{l}\text { The system is simple and flexible. } \\
\text { The communication between the sensor nodes is wired. } \\
\text { - } \quad \text { Less costly system. } \\
\text { The system used valid for interior parking. }\end{array}$ & $\begin{array}{l}\text { Linear networking is used. } \\
\text { The system uses a display with a limited web application. } \\
\text { The system misses him the remote monitoring of the availability of } \\
\text { available spaces in the car park. }\end{array}$ \\
\hline S3: [7] & $\begin{array}{l}\text { Interior parking, } \\
\text { linear, no adaptable, } \\
\text { single area }\end{array}$ & $\begin{array}{l}\text { Less expensive system. } \\
\text { The GSM system used to reserve free parking spaces. } \\
\text { The proposed system is valid for private indoor car parks with a gate } \\
\text { and an entry and exit barrier. }\end{array}$ & $\begin{array}{l}\text { The system is limited for an improvement in the future in the } \\
\text { implementation of the latter in outdoor linear car parks. } \\
\text { No mobile application is used for tracking vacant spaces remotely. } \\
\text { The GSM system used for reservation of spaces which can be blocked } \\
\text { with multiple access. }\end{array}$ \\
\hline S4: [8] & $\begin{array}{l}\text { Interior parking, } \\
\text { linear, no adaptable, } \\
\text { single area }\end{array}$ & $\begin{array}{l}\text { - } \quad \text { System robust to communication interference between different nodes. } \\
\text { - } \quad \text { Hybrid system with RFID and wireless sensor. } \\
\text { RFID system for predefined parking. }\end{array}$ & $\begin{array}{l}\text { - The system lacks him an application for the remote monitoring of } \\
\text { free spaces. } \\
\text { No navigation system or additional services to increase the scalability of } \\
\text { the latter. } \\
\text { The topology used is wired bus which limits the interoperability and the } \\
\text { scalability of the system. }\end{array}$ \\
\hline S5: [9] & $\begin{array}{l}\text { Outdoor parking, } \\
\text { Linear, no adaptable, } \\
\text { single area }\end{array}$ & $\begin{array}{ll}\text { - } & \text { Cloud system based on Google to manage mobile applications. } \\
\text { - } & \text { Use of NFC payment system. } \\
\text { - } & \text { Outdoor navigation based on the GOOGLE API. } \\
\text { - } & \text { Management of spaces in an efficient way. }\end{array}$ & $\begin{array}{l}\text { The outdoor parking system which is valid only for linear car parks and } \\
\text { not for mass car parks. } \\
\text { The payment is not checked. } \\
\text { - Use of RFID only for certain parking spaces. }\end{array}$ \\
\hline S6: [10] & $\begin{array}{l}\text { Interior parking, mass, } \\
\text { no adaptable, single } \\
\text { area }\end{array}$ & $\begin{array}{l}\text { - } \quad \text { System based on ZigBee technology in the transmission of detected data. } \\
\text { Internal LCD-based guidance system. } \\
\text { The system is a mass car park that uses the cluster architecture in } \\
\text { self-organization of the WSN. } \\
\quad \text { Flexible and less expensive system. }\end{array}$ & $\begin{array}{l}\text { The system is not adaptable to linear parking. } \\
\text { The system lacks him a navigation mechanism or an external } \\
\text { guidance system. } \\
\text { No payment system is implemented in this system. }\end{array}$ \\
\hline S7: [11] & $\begin{array}{l}\text { Interior parking, mass, } \\
\text { no adaptable, } \\
\text { multiarea }\end{array}$ & $\begin{array}{l}\text { - The driver can know the empty spaces in the parking lot. } \\
\text { - The system encompasses several car parks scattered throughout the city. } \\
\text { Drivers can detect parking spaces using a smartphone application. }\end{array}$ & $\begin{array}{l}\text { - Light sensors are sensitive to detect the cars presence. } \\
\text { The system used is valid for private outdoor car parks and not } \\
\text { for public. } \\
\text { The system has no navigation system to the car parks available in } \\
\text { the city. } \\
\text { The driver cannot make remote payment. }\end{array}$ \\
\hline
\end{tabular}


Table 2. Cont.

\begin{tabular}{|c|c|c|c|}
\hline $\begin{array}{c}\text { Smart Parking } \\
\text { Systems }\end{array}$ & $\begin{array}{c}\text { Type of Parking } \\
\text { System }\end{array}$ & Advantages & Disadvantages \\
\hline S8: [12] & $\begin{array}{l}\text { Interior parking, mass, } \\
\text { no adaptable, single } \\
\text { area }\end{array}$ & $\begin{array}{ll}\text { - } & \text { The use of RFID to identify drivers and realize payment. } \\
\text { - } & \text { Less expensive system. } \\
\text { - } & \text { System is valid for indoor car parks. } \\
& \text { The guiding system used indoors based on LCD screens. }\end{array}$ & $\begin{array}{l}\text { The system is valid only to mass parking. } \\
\text { No application is developed to facilitate the task for drivers when } \\
\text { searching of available spaces. }\end{array}$ \\
\hline S9: [13] & $\begin{array}{l}\text { Outdoor parking, } \\
\text { mass, no adaptable, } \\
\text { single area }\end{array}$ & $\begin{array}{ll}\text { - } & \text { The system is based on sending data over IPv6. } \\
\text { - } & \text { RFID system is deployed. } \\
\text { Anti-theft system based on sending GSM messages to the police. } \\
\text { - The use of hybrid sensors in the indoor parking using barriers to } \\
\text { manage inputs and outputs. }\end{array}$ & $\begin{array}{l}\text { - The use of IR sensors that are sensitive to vehicle lights. } \\
\text { - } \quad \text { No security system at the exit. } \\
\text { No guidance or navigation system is used. }\end{array}$ \\
\hline S10: [14] & $\begin{array}{l}\text { Outdoor parking, } \\
\text { mass, no adaptable, } \\
\text { multiarea }\end{array}$ & $\begin{array}{l}\text { - The system uses the tree topology for the deployment of sensors in the } \\
\text { outdoor car parks. } \\
\text { - Mobile application for drivers and agents. } \\
\text { - The agents have an important role in monitoring the parking spaces in } \\
\text { each area. } \\
\text { Using LEDs to display the number of empty spaces in each area. }\end{array}$ & - The system used is valid only for linear outdoor car parks. \\
\hline S11: [15] & $\begin{array}{l}\text { Interior parking, mass, } \\
\text { no adaptable, single } \\
\text { area }\end{array}$ & $\begin{array}{l}\text { - } \quad \text { Outdoor navigation implemented. } \\
\text { The security and payment system based on RFID. } \\
\text { The use of an application based on Google-MAP to locate all available } \\
\text { parking spaces. }\end{array}$ & $\begin{array}{l}\text { The system is valid only for interior car parks. } \\
\text { The application is limited for the visualization of the free spaces in } \\
\text { the interior. }\end{array}$ \\
\hline S12: [16] & $\begin{array}{l}\text { Interior parking, mass, } \\
\text { no adaptable, single } \\
\text { area }\end{array}$ & $\begin{array}{l}\text { - The use RFID and ALPR for the identification and the security. } \\
\text { - The use an application mobile to navigate to parking area and to locate } \\
\text { available spaces. } \\
\text { The use NFC for online payment. }\end{array}$ & $\begin{array}{l}\text { - The system is valid only to mass parking. } \\
\text { - } \quad \text { The topology created by the network is fixed. }\end{array}$ \\
\hline S13: [17] & $\begin{array}{l}\text { Outdoor parking, no } \\
\text { adaptable, multiarea }\end{array}$ & $\begin{array}{ll}\text { - } & \text { The use of powerful sensors. } \\
\text { - } & \text { The use of a mobile application (ParkX) to navigate to parking spaces. } \\
\text { - } & \text { The payment of parking fees is implemented. }\end{array}$ & $\begin{array}{ll}\text { - } & \text { The sensors used are expensive. } \\
\text { - } & \text { No security system is implemented. } \\
\text { - } & \text { oooking in advance can create parking problems. }\end{array}$ \\
\hline S14: [18] & $\begin{array}{l}\text { Outdoor-indoor } \\
\text { parking, no adaptable, } \\
\text { single area }\end{array}$ & $\begin{array}{l}\text { - } \quad \text { The parking entrance and exit management is implemented using RFID. } \\
\text { The security and the spaces management are based on RFID. }\end{array}$ & $\begin{array}{l}\text { - } \quad \text { The use Wi-Fi multi-hop communication that consumes too much. } \\
\text { - No guidance or navigation system is used. } \\
\text { - No mobile application is used. }\end{array}$ \\
\hline Proposed system & $\begin{array}{l}\text { Outdoor-indoor } \\
\text { parking, adaptable, } \\
\text { multiarea }\end{array}$ & $\begin{array}{l}\text { Topology adaptable to any type of parking. } \\
\text { The use an application mobile to navigate to parking area and to locate } \\
\text { available spaces. } \\
\text { RFID system is deployed. } \\
\text { - Security and Reservation system is deployed. } \\
\text { - The use of the online payment system. }\end{array}$ & \\
\hline
\end{tabular}


Table 3. Different technologies and services used in smart parking systems.

\begin{tabular}{|c|c|c|c|c|c|c|c|c|c|c|c|c|}
\hline \multirow{2}{*}{ System: Ref } & \multicolumn{2}{|c|}{ Communication Technology } & \multicolumn{7}{|c|}{ Services } & \multicolumn{3}{|c|}{ Connection Access } \\
\hline & TS & TD & GD & PY & SE & RE & SG & AV & PS & IoT & SM & WA \\
\hline S1: [5] & & GSM & & & $\sqrt{ }$ & $\sqrt{ }$ & $\sqrt{ }$ & $\sqrt{ }$ & & & & $\sqrt{ }$ \\
\hline S2: [6] & ZigBee & & $\sqrt{ }$ & & & & & $\sqrt{ }$ & & & & $\sqrt{ }$ \\
\hline S3: [7] & ZigBee & GSM & & $\sqrt{ }$ & $\sqrt{ }$ & $\sqrt{ }$ & & & $\sqrt{ }$ & & $\sqrt{ }$ & \\
\hline S4: [8] & & Bluetooth & & $\sqrt{ }$ & $\sqrt{ }$ & & & & $\sqrt{ }$ & & $\sqrt{ }$ & \\
\hline S5: [9] & ZigBee & $\mathrm{Wi}-\mathrm{Fi} / 3 \mathrm{G}$ & $\sqrt{ }$ & $\sqrt{ }$ & & & $\sqrt{ }$ & $\sqrt{ }$ & & $\sqrt{ }$ & $\sqrt{ }$ & \\
\hline S6: [10] & & & $\sqrt{ }$ & & & & & & & & & \\
\hline S7: [11] & Wi-Fi & $\mathrm{Wi}-\mathrm{Fi} / 3 \mathrm{G}$ & & & & & & $\sqrt{ }$ & $\sqrt{ }$ & & $\sqrt{ }$ & $\sqrt{ }$ \\
\hline S8: [12] & ZigBee & & $\sqrt{ }$ & & & & $\sqrt{ }$ & & & & & \\
\hline S9: [13] & & & & $\sqrt{ }$ & & & $\sqrt{ }$ & $\sqrt{ }$ & $\sqrt{ }$ & $\sqrt{ }$ & $\sqrt{ }$ & $\sqrt{ }$ \\
\hline S10: [14] & ZigBee & $\mathrm{Wi}-\mathrm{Fi} / 3 \mathrm{G}$ & $\sqrt{ }$ & & $\sqrt{ }$ & & $\sqrt{ }$ & $\sqrt{ }$ & $\sqrt{ }$ & $\sqrt{ }$ & $\sqrt{ }$ & \\
\hline S11: [15] & & & & $\sqrt{ }$ & & $\sqrt{ }$ & & & $\sqrt{ }$ & & $\sqrt{ }$ & \\
\hline S12: [16] & ZigBee & & & $\sqrt{ }$ & & $\sqrt{ }$ & & $\sqrt{ }$ & $\sqrt{ }$ & & $\sqrt{ }$ & \\
\hline S13: [17] & Wi-Fi & & $\sqrt{ }$ & $\sqrt{ }$ & & $\sqrt{ }$ & $\sqrt{ }$ & $\sqrt{ }$ & & $\sqrt{ }$ & $\sqrt{ }$ & \\
\hline S14: [18] & Wi-Fi & & & & $\sqrt{ }$ & & & & & & & \\
\hline Proposed system & ZigBee & $\mathrm{Wi}-\mathrm{Fi} / 3 \mathrm{G}$ & $\sqrt{ }$ & $\sqrt{ }$ & $\sqrt{ }$ & $\sqrt{ }$ & $\sqrt{ }$ & $\sqrt{ }$ & $\sqrt{ }$ & $\sqrt{ }$ & $\sqrt{ }$ & $\sqrt{ }$ \\
\hline
\end{tabular}

TS: technology used by sensor network; TD: technology used by the drivers; GD: guidance; PY: payment; SE: security; RE: reservation; IG: smart gateway; PS: parking management using smartphone; AV: availability checking over internet; IOT: Internet of Things; WA: web application; SM: smartphone; $\sqrt{ }$ : the technology or the service is used in the paper.

\section{Conclusions}

In this paper, we realised a thorough comparative study of different architectures and the different self-organization protocols used in the management of the different types of existing car parks in the cities. We have also proposed a new architecture of a new smart parking system based on the deployment and implementation of different technologies, such as WSN, IoT, RFID. This new architecture implements, on the one hand, a new hybrid and adaptable self-organization protocol for the deployment of sensor nodes in different environments in order to maximize the performance of the WSN and increase its longevity, and, on the other hand, uses existing technologies, such as WSN and RFID to minimize the cost of implementing the system and improve the quality of its design.

In future work, we will detail and develop this new adaptable self-organization protocol for wireless sensor networks by performing simulations in order to demonstrate its strength by comparing it with other existing self-organization protocols.

Author Contributions: The work presented here was carried out in collaboration between all authors. Adil Hilmani and Abderrahim Maizate contributed in the design and development of the smart parking system; Adil Hilmani wrote the paper. Aberrahim Maizate and Larbi Hassouni supervised the project.

Acknowledgments: The authors would like to thank the editor and anonymous referees for their valuable comments to improve the quality of this paper.

Conflicts of Interest: The authors declare no conflicts of interest.

\section{References}

1. Kalantary, S.; Taghipour, S. A survey on architectures, protocols, applications, and management in wireless sensor networks. J. Adv. Comput. Sci. Technol. 2014, 3, 1. [CrossRef]

2. Akyildiz, I.F.; Vuran, M.C. Wireless Sensor Networks; Wiley Publication: Hoboken, NJ, USA, 2010.

3. Akyildiz, I.F.; Su, W.; Sankarasubramaniam, Y.; Cayirci, E. A survey on sensor networks. IEEE Commun. Mag. 2002, 40, 102-114. [CrossRef]

4. Hilmani, A.; Maizate, A. A study of self-organization protocols in wireless sensor network. Mediterr. Telecommun. J. 2017, 7. N².

5. Rahayu, Y.; Mustapa, F.N. A secure parking reservation system using GSM technology. Int. J. Comput. 2013, 2, 518. [CrossRef]

6. Yee, H.C.; Rahayu, Y. Monitoring parking space availability via ZigBee technology. Int. J. Future Comput. 2014, 3, 377 . 
7. Poojaa, A.; Glory, M.; Nathiya, P.; Ramya, R.; Sivasrinee, E.T. WSN based secure vehicle parking management and reservation system. In Proceedings of the National Conference on Research Advances in Communication, Computation, Electrical Scienceand Structures (NCRACCESS-2015), Deviyakurichi, India, 21 February 2015.

8. Karbab, E.M.; Djenouri, D.; Boulkaboul, S. Car park management with networked wireless sensors and active RFID. In Proceedings of the IEEE International Conference on Electro/Information Technology (EIT), Dekalb, IL, USA, 21-23 May 2015.

9. Mainetti, L.; Palano, L.; Patrono, L.; Stefanizzi, M.L.; Vergallo, R. Integration of RFID and WSN technologies in a smart parking system. In Proceedings of the 22nd International Conference on Software, Telecommunications and Computer Networks (SoftCOM), Split, Croatia, 17-19 September 2014.

10. Chen, M.; Chang, T. A parking guidance and information system based on wireless sensor network. In Proceedings of the IEEE International Conference on Information and Automation, Shenzhen, China, 6-8 June 2011.

11. Yang, J.; Portilla, J.; Riesgo, T. Smart parking service based on Wireless Sensor Networks. In Proceedings of the IECON 2012 - 38th Annual Conference on IEEE Industrial Electronics Society, Montreal, QC, Canada, 25-28 October 2012.

12. Patil, M.; Bhonge, V.N. Wireless sensor network and RFID for smart parking system. Int. J. Emerg. Technol. Adv. Eng. 2013, 3, 188-192.

13. Gandhi, B.M.K.; Rao, M.K. A prototype for IoT based car parking management system for smart cities. Indian J. Sci. Technol. 2016, 9. [CrossRef]

14. Quiñones, M.; Gonazález, V.; Quiñones, L. Design of a smart parking system using wireless sensor network. In Proceedings of the 10th Iberian Conference on Information Systems and Technologies (CISTI), Aveiro, Portugal, 17-20 June 2015.

15. Orrie, O.; Silva, B.; Hancke, G.P. A wireless smart parking system. In Proceedings of the IECON 2015-41st Annual Conference of the IEEE Industrial Electronics Society, Yokohama, Japan, 9-12 November 2015.

16. Chandra, H.; Hadisaputra, K.R.; Santoso, H.; Anggadjaja, E. Smart Parking Management System: An integration of RFID, ALPR, and WSN. In Proceedings of the IEEE 3rd International Conference on Engineering Technologies and Social Sciences (ICETSS), Bangkok, Thailand, 7-8 August 2017.

17. Gupta, A.; Kulkarni, S.; Jathar, V.; Sharma, V.; Jain, N. Smart Car Parking Management System Using IoT. Am. J. Sci. Eng. Technol. 2017, 2, 112-119.

18. Tsiropoulou, E.E.; Baras, J.S.; Papavassiliou, S.; Sinha, S. RFID-based smart parking management system. Cyber-Phys. Syst. 2017, 1-20. [CrossRef]

19. Wagh, S.S.; More, A.; Kharote, P.R. Performance Evaluation of IEEE 802.15.4 Protocol under Coexistence of WiFi 802.11b. Procedia Comput. Sci. 2015, 57, 745-751. [CrossRef]

20. Olasupo, T.O.; Otero, C.E.; Otero, L.D.; Olasupo, K.O.; Kostanic, I. Path Loss Models for Low-Power, Low-Data Rate Sensor Nodes for Smart Car Parking Systems. IEEE Trans. Intell. Transp. Syst. 2017. [CrossRef]

21. Shuaib, K.; Alnuaimi, M.; Boulmalf, M.; Jawhar, I.; Sallabi, F.; Lakas, A. Performance Evaluation of IEEE 802.15.4: Experimental and Simulation Results. J. Commun. 2007, 2, 29-37. [CrossRef]

(C) 2018 by the authors. Licensee MDPI, Basel, Switzerland. This article is an open access article distributed under the terms and conditions of the Creative Commons Attribution (CC BY) license (http:/ / creativecommons.org/licenses/by/4.0/). 